\title{
Distance Browsing in Distributed Multimedia Databases
}

\author{
Fabrizio Falchi \\ ISTI-CNR \\ Pisa, Italy \\ fabrizio.falchi@isti.cnr.it \\ Fausto Rabitti \\ ISTI-CNR \\ Pisa, Italy \\ fausto.rabitti@isti.cnr.it
}

\author{
Claudio Gennaro \\ ISTI-CNR \\ Pisa, Italy \\ claudio.gennaro@isti.cnr.it \\ Pavel Zezula \\ Masaryk University \\ Brno, Czech Republic \\ zezula@fi.muni.cz
}

\begin{abstract}
The state of the art of searching for non-text data (e.g., images) is to use extracted metadata annotations or text, which might be available as a related information. However, supporting real content-based audio-visual search, based on similarity search on features, is significantly more expensive than searching for text. Moreover, such search exhibits linear scalability with respect to the data set size, so parallel query execution is needed.

In this paper, we present a Distributed Incremental Nearest Neighbor algorithm $(D I N N)$ for finding closest objects in an incremental fashion over data distributed among computer nodes, each able to perform its local Incremental Nearest Neighbor (local-INN) algorithm. We prove that our algorithm is optimum with respect to both the number of involved nodes and the number of local-INN invocations. An implementation of our DINN algorithm, on a real P2P system called $M C A N$, was used for conducting an extensive experimental evaluation on a real-life dataset.

The proposed algorithm is being used in two running projects: SAPIR and NeP4B.
\end{abstract}

\section{INTRODUCTION}

A large component of the Web content nowadays consists of non-text data, such as images, music, animations, and videos. Current search engines index Web documents by their textual content. For instance, web tools for performing image searching (such as the ones provided by Google, Yahoo!, or MSN Live Search) simply index the text associated with the image and the ALT attribute of the IMG tag used to provide a description of an image.

Image indexing methods based on content-based analysis or pattern matching (which for instance analyzes the characteristics of images, i.e., features, such as colors and shapes) are usually not exploited at all. The problem is that these processes are significantly more expensive than text analysis. Nevertheless, what is more important is that the search on the level of features exhibits linear scalability with respect to the data search size, which is not acceptable for the expected dimension of the problem. The reason is that for this kind of data the appropriate search methods are based on similarity paradigms that typically exploits range queries and nearest neighbor queries. These queries are computationally more intensive than the exact match, because conventional inverted indexes used for text are not applicable for such data.
Besides multimedia information retrieval, there are other applications, such as bioinformatics, data mining, pattern recognition, machine learning, computer vision, that can take advantage of the similarity search paradigm. However, different applications have in general different similarity functions. A convenient way to address this problem and achieve one solution for several purposes is to formalize the similarity by the mathematical notion of the metric space. Here data elements are assumed to be objects from a metric space where pairwise distances between the objects can be determined and where any distance satisfies the properties of symmetry, non-negativity, identity, and triangle inequality [16]. In this respect, the metric space approach to similarity searching is highly extensible. However, our Distributed Incremental Nearest Neighbor (DINN) algorithm does even not require the objects to be metric - we only suppose that the distance is non-negative.

To address the problems of scalability, P2P communication paradigm seems to be a convenient approach, and several scalable and distributed search structures have been proposed even for the most generic case of metric space searching (see [3] and [4]) for a survey). A common characteristic of all these existing approaches is the autonomy of the peers with no need of central coordination or flooding strategies. Since there are no bottlenecks, the structures are scalable and high performance is achieved through parallel query execution on individual peers.

Since the number of closest objects is typically easier to specify than establishing a search range, users prefer the nearest neighbors to the range queries. For example, given an image, it is easier to ask for 10 most similar ones according to an image similarity criterion than to define the similarity threshold quantified as a real number. However, nearest neighbors algorithms are typically more difficult to implement, and in $\mathrm{P} 2 \mathrm{P}$ environments the situation is even worse. The main reason is that traditional (optimum) approaches [10] are based on a priority queue with a ranking criterion, which sequentially decides the order of accessed data buckets. In fact, the existence of centralized entities and sequential processing are completely in contradiction with decentralization and parallelism objectives of any P2P search network. Things are further complicated by the natural necessity of some applications to retrieve the nearest neighbor in an incremental fashion, because the number of desired neighbors is unknown in advance. By incremental, we mean that such an algorithm computes the neighbors one 
by one, without the need to re-compute the query from the scratch.

An important example of application of Incremental Nearest Neighbor is processing of complex queries, that is queries involving more than one feature overlay, such as: find all images most similar to the query image with respect to the color and the shape at once. In this situation, we do not know how many neighbors must be retrieved in individual layers before the best object is found that satisfies the complex condition. In fact, the widely used $A_{0}$ (also called Fagin's Algorithm) [5] as well as the threshold algorithm [6] suppose that each single source for a specific feature is able to perform a $I N N$ algorithm.

In this paper, we present a first attempt to approach the Incremental Nearest Neighbor problem for P2P-based systems. Our proposed solution, based on a generalization of the algorithm proposed in [10] for hierarchical centralized structures, is optimal and independent from any specific P2P architecture - it can be applied to any Scalable and Distributed Data Structure (SDDS), P2P system, and Gridbased similarity search infrastructure. We implemented our algorithm on a real $\mathrm{P} 2 \mathrm{P}$ system called $M C A N[8,9]$ and we conducted an extensive experimental evaluation on a reallife dataset of $1,000,000$ objects. $M C A N$ is a scalable distributed similarity search structure for metric data (for a survey see [3]) which extends the Content-Addressable Network (CAN) (a well known Distributed Hash Table).

The INN algorithm is being used in two running projects: SAPIR $^{1}$ and NeP4B ${ }^{2}$. The European project SAPIR (Search on Audio-visual content using Peer-to-peer Information Retrieval) aims at finding new ways to analyze, index, and retrieve the tremendous amounts of speech, image, video, and music that are filling our digital universe, going beyond what the most popular engines are still doing, that is, searching using text tags that have been associated to multimedia files. SAPIR is a three-year research project that aims at breaking this technological barrier by developing a large-scale, distributed peer-to-peer infrastructure that will make it possible to search for audio-visual content by querying the specific characteristics (i.e. features) of the content. SAPIR's goal is to establish a giant peer-to-peer network, where users are peers that produce audiovisual content using multiple devices (e.g., cell phones) and service providers will use more powerful peers that maintain indexes and provide search capabilities

NeP4B (Networked Peers for Business), is an Italian project aiming at innovative ICTs solutions for Small and Medium size Enterprizes (SMEs), by developing an advanced technological infrastructure to enable companies of any nature, size and geographic location to search for partners, negotiate and collaborate without limitations and constraints. The infrastructure will base on independent and interoperable semantic peers which behave as nodes of a virtual network. The project vision is towards an Internet-based structured marketplace where companies can access the huge amount of information already present in vertical portals and corporate databases and use it for dynamic, value-adding collaboration purposes. In the $\mathrm{NeP} 4 \mathrm{~B} \mathrm{P} 2 \mathrm{P}$ infrastructure the semantic peers represent aggregations of SMEs with similar activities and the multimedia objects are descriptions/presentations of

\footnotetext{
${ }^{1}$ http://www.sapir.eu/

${ }^{2}$ http://dbgroup.unimo.it/nep4b/
}

their products/services extracted from the companies' Web sites.

The rest of the paper is organized as follows. Section 2 presents the related work. Section 3 provides an overview of our proposed solution while the formal definition is given in Section 4. In Section 5 we report the results of an extensive experimental evaluation of the DINN over the MCAN. Conclusions and future work are discussed in Section 6 .

An earlier version of this paper has been presented at the Second International Conference on Scalable Information Systems (INFOSCALE 2007) [7].

\section{RELATED WORK}

Our proposed solution is based on a generalization of the algorithm proposed in [10]. The incremental nearest neighbor algorithm defined in [10] is applicable whenever the search space is structured in a hierarchical manner. The algorithm starts off by initializing the queue of pending requests with the root of the search structure - since the order of entries in this queue is crucial, they refer to it as the priority queue. In the main loop, the element closest to the query is taken off the queue. If it is an object, it reports it as the next nearest object. Otherwise, the child elements of the element in the search hierarchy are inserted into the priority queue.

In [15] an efficient algorithm to perform $k-N N$ in a $\mathrm{P} 2 \mathrm{P}$ system (specifically the Chord [14]) is proposed. The algorithm uses the same priority queue based approach of [10]. As far as we know, it is the first attempt to extend [10] to the distributed environment making use of the parallelism of the P2P network.

They define their algorithm for a hierarchical index (as in [10]). To provide distributed hashing of spatial data they use a distributed quadtree index they developed, although they say that other indices can be utilized as well (e.g., P2P $\mathrm{R}$-trees [11]). The query is first initiated on a single peer in the $\mathrm{P} 2 \mathrm{P}$ network. This peer maintains the priority queue of quadtree blocks (mapping to a control point each) that are being processed for the query. To process a block, they have to contact from this query initiating peer, the peer that owns that block, i.e., the control point. Hence, in their parallel algorithm, they contact, rather than just the top entry of the priority queue, a multiple number of these peers.

\section{DINN OUTLINE}

The INN algorithm [10] was defined for a large class of centralized hierarchical spatial data structures. Instead our $D I N N$ algorithm is distributed and not limited to hierarchical structures. Thus it can be used over SDDSs, P2P systems and Grid infrastructures. Our algorithm is built over nodes which are able to perform locally an $I N N$ between the objects they store (this will be formalized in Assumption 1).

In particular, we reformulate the definition of priority queue (Queue) given in [10] by considering as elements of Queue, objects and nodes (or peers). We prove that our algorithm is optimal, in terms of both number of involved nodes and local-INN invocations. The elements of Queue are ordered according to a key which is always associated with both objects and nodes. The key associated with each object is the distance between the query and the object itself. Instead the key associated with each node is a lower bound for the distance between the query and the next re- 
sult coming from the node. While for an already involved node this lower bound can be simply the distance from the query of the last object retrieved by its local-INN, for the not yet involved nodes a naive solution could be to always use 0 as lower bound. However, this would imply all nodes to be involved for every similarity query. To avoid this, we suppose that each node is able to evaluate this lower bound for every node it knows (in $\mathrm{P} 2 \mathrm{P}$ systems they are called neighbors).

Furthermore, in $\mathrm{P} 2 \mathrm{P}$ systems there is no global knowledge of the network. Thus, we make an assumption (see Assumption 2) regarding the ability to find the next most promising node (by considering the lower bound mentioned before). This assumption replace the consistency condition used in [10] for hierarchical data structures. We prove that our assumption can be satisfied under one of two simpler conditions (see Subsection 4.3.3) which are common for data structures able to perform similarity search.

During the DINN algorithm execution, Queue contains a certain number of entries sorted in order of decreasing key. Entries can be both nodes and objects. Because of the values used as key, when a node is after an object we are sure that no better results than the object itself can be found in the node. The algorithm proceeds by processing Queue from the top. Basically if the first entry of the queue is an object, this object is the result of the DINN. In case the first entry is a node, we invoke its local-INN. The resulting object of this invocation is placed in Queue and its distance from the query allows us to update the entry with a more accurate (greater) lower bound which moves the node backward in Queue.

This outlined implementation is intrinsically sequential, since a single step of the algorithm involves only the first element of Queue at a time. In the second part of the paper, we straightforwardly generalize the algorithm introducing parallelism by invoking the local-INN algorithm of more than one node simultaneously. The precise definition of the algorithms is provided in the next section. Examples are given to help understanding the algorithm.

\section{THE DINN ALGORITHM}

\subsection{Definitions and Notation}

In this subsection we provide a number of definitions and notations required to define the $D I N N$ algorithm.

Notation:

- $\mathcal{N}$ is the set of the nodes participating in the distributed system

- $\mathcal{D}$ is the objects domain

- $\mathcal{X}_{i} \subset \mathcal{D}$ is the set of the objects stored in a given node $N_{i} \in \mathcal{N}$

- $\mathcal{X}=\bigcup_{i} \mathcal{X}_{i}$ is the set of the objects stored in the whole network.

As in [10], our DINN is based on a priority queue:

Definition 1. A priority queue (Queue) is a set of pairs $\langle$ element, $\vartheta\rangle$ ordered according to key $\vartheta \in \mathbb{R}^{+}$. An element can be either an object or a node.

\begin{tabular}{|c|c|}
\hline Symbol & Meaning \\
\hline$\overline{\mathcal{N}}$ & $\begin{array}{l}\text { the set of the nodes participating in the dis- } \\
\text { tributed system }\end{array}$ \\
\hline$N_{i}$ & $\begin{array}{l}\text { a node participating in the distributed system } \\
\left(N_{i} \in \mathcal{N}\right)\end{array}$ \\
\hline$\overline{\mathcal{D}}$ & the objects domain \\
\hline$x$ & an object in the domain $(x \in \mathcal{D})$ \\
\hline$d(x, y)$ & the distance between $x$ and $y(x, y$ \\
\hline$r$ & a value in $\mathbb{R}^{+}$ \\
\hline $\mathcal{X}_{i}$ & $\begin{array}{l}\mathcal{X}_{i} \subset \mathcal{D} \text { is the set of the objects stored in a } \\
\text { given node } N_{i} \in \mathcal{N}\end{array}$ \\
\hline $\bar{X}$ & $\begin{array}{l}\text { the set of the objects stored in the whole net- } \\
\text { work }\left(\mathcal{X}=\bigcup_{i} \mathcal{X}_{i}\right)\end{array}$ \\
\hline$\delta\left(N_{i}, x\right)$ & $\begin{array}{l}\text { the lower bound for the distances between } x \in \\
\mathcal{D} \text { and all the objects stored in } N_{i} \text { (i.e., } \mathcal{X}_{i} \text { ) }\end{array}$ \\
\hline $\mathcal{N}_{x, r}$ & $\begin{array}{l}\text { the set of nodes in } \mathcal{N} \text { that could have objects } \\
\text { closer to } x \text { than } r\end{array}$ \\
\hline $\mathcal{N}_{x}$ & $\begin{array}{l}\text { a subset of nodes } \mathcal{N} \text { which is either downward } \\
\text { closed (with respect to } x \text { ) or empty }\end{array}$ \\
\hline$N_{n}$ & the closest node to $x$ in $\left(\mathcal{N} \backslash \mathcal{N}_{x}\right)$ \\
\hline$\overline{\mathcal{N}}^{*}$ & $\begin{array}{l}\text { the set of nodes that already performed a local- } \\
\text { INN }\end{array}$ \\
\hline$e$ & $\begin{array}{l}\text { an element, either an object or a node, in } \\
\text { Queue }\end{array}$ \\
\hline$\vartheta$ & $\begin{array}{l}\vartheta \in \mathbb{R}^{+} \text {is the key used for ordering the ele- } \\
\text { ments of Queue }\end{array}$ \\
\hline Queue & $\begin{array}{l}\text { the set of pairs }\langle\text { element, } \vartheta\rangle \text { ordered according } \\
\text { to key } \vartheta\end{array}$ \\
\hline $\bar{k}$ & $\begin{array}{l}\text { the number of objects already retrieved by the } \\
\text { previous invocations of the } D I N N\end{array}$ \\
\hline$k^{+}$ & $\begin{array}{l}\text { the number of next neighbors we want to re- } \\
\text { trieve }\end{array}$ \\
\hline$k_{a n s}$ & $\begin{array}{l}\text { the number of results already found by the } \\
D I N N \text { during the current invocation }\end{array}$ \\
\hline$\hat{k}$ & $\hat{k}=k^{+}-k_{a n s}$ \\
\hline$x_{\hat{k}}$ & $x_{\hat{k}} \in \mathcal{X}$ is the $\hat{k}$-th object in Queue \\
\hline$p$ & $\begin{array}{l}p \in[0,1] \text { is the parameter used to set the de- } \\
\text { gree of parallelization of the } D I N N\end{array}$ \\
\hline$l_{i}$ & ed by $N_{i}$ \\
\hline
\end{tabular}

\section{Table 1: Notation}

In order to avoid involving all the nodes in the DINN execution, we suppose there is the possibility to evaluate a lower bound $(\delta)$ for the distances between the objects stored in a certain node and any given object in $\mathcal{D}$.

Definition 2. Given a node $N_{i} \in \mathcal{N}$ and an object $x \in \mathcal{D}$ we define $\delta: \mathcal{N} \times \mathcal{D} \rightarrow \mathbb{R}^{+}$as a lower bound for the distances between $x$ and all the objects stored in $N_{i}$ (i.e., $\mathcal{X}_{i}$ ):

$$
\delta\left(N_{i}, x\right) \leq \min \left\{d(y, x), y \in \mathcal{X}_{i}\right\}
$$

Note that this lower bound could even be 0 for every node. Thus we do not strictly require this lower bound to be evaluable, but we use it for efficiency in case it can be given. In case each node $N_{i} \in \mathcal{N}$ of a given distributed data structure is responsible for a portion $\mathcal{D}_{i}$ of the domain $\mathcal{D}$ we will say that $\delta$ is strong iff:

$$
\forall N_{i} \in \mathcal{N}, \delta\left(N_{i}, x\right)=0 \Leftrightarrow x \in \mathcal{D}_{i}
$$


In defining our DINN algorithm we will use the general notion of downward closed set. We will limit this notion to set of nodes with respect to a given object (making use the lower bound $\delta$ defined above).

Definition 3. A set of nodes $\mathcal{N}_{x}$ is downward closed with respect to an object $x \in \mathcal{D}$ iff $\forall N_{j}, N_{i} \in \mathcal{N}$ :

$$
N_{i} \in \mathcal{N}_{x} \wedge \delta\left(N_{j}, x\right)<\delta\left(N_{i}, x\right) \Rightarrow N_{j} \in \mathcal{N}_{x}
$$

In other words, if a set of nodes is downward closed with respect to an object $x \in \mathcal{D}$, there are no nodes, out of the set, with a lower bound less to those of the nodes in the set. In Figure 1 we give an example of downward closed sets of nodes. The position of each node $N_{i}$ on the axis is determined by $\delta\left(N_{i}, x\right)$. The nodes are grouped in all the possible downward closed sets. Note that, by Definition 3, each set contains $N_{1}$ which has the minimum $\delta$ from $x$. Moreover, each downward closed set of nodes contains any node between $N_{1}$ and the furthest away node in the set itself. Obviously, the position on the axis of the nodes depends on the particular object $x$.

Another special set of nodes we will refer in the algorithm definition is the set of nodes whose lower bound $\delta$ is less than a given $r \in \mathbb{R}^{+}$:

Definition 4. Let $x$ be an object in $\mathcal{D}$ and $r \in \mathbb{R}^{+} . \mathcal{N}_{x, r}$ is the set of nodes in $\mathcal{N}$ that could have objects closer to $x$ than $r$, i.e.,

$$
\mathcal{N}_{x, r}=\left\{N_{i}: N_{i} \in \mathcal{N} \wedge \delta\left(N_{i}, x\right) \leq r\right\} .
$$

In Table 4.1, we report the list of symbols used in this paper with their corresponding meaning. Please note that in this table also symbols that will be defined and used in the next section can be found.

\subsection{Assumptions}

Our DINN algorithm is based on two assumptions.

Assumption 1. Each node $N_{i} \in \mathcal{N}$ is able to perform a local-INN algorithm over the objects $\mathcal{X}_{i} \subseteq \mathcal{D}_{i}$ it stores.

Assumption 2. Let $x \in \mathcal{D}$ be an object in the domain. Let $\mathcal{N}_{x} \subseteq \mathcal{N}$ be a subset of nodes which is either downward closed (with respect to $x$ ) or empty. Let $N_{n} \in\left(\mathcal{N} \backslash \mathcal{N}_{x}\right)$ be the closest node to $x$ in $\left(\mathcal{N} \backslash \mathcal{N}_{x}\right)$, i.e.,

$$
N_{n}=\arg \min _{N_{i}}\left\{\delta\left(N_{i}, x\right), N_{i} \in\left(\mathcal{N} \backslash \mathcal{N}_{x}\right)\right\}
$$

Whenever an arbitrary node $N_{c} \in \mathcal{N}$ knows $\mathcal{N}_{x}$ (i.e., would be able to contact all the nodes in $\left.\mathcal{N}_{x}\right), N_{c}$ must be able to check if $N_{n}$ exists (i.e., $\left.\left(\mathcal{N} \backslash \mathcal{N}_{x}\right) \neq \emptyset\right)$ and, eventually, to contact it.

Assumption 1 is needed because our DINN algorithm is built over nodes which are able to perform a local-INN.

Assumption 2 is necessary for engaging the nodes in the $D I N N$ algorithm execution as it progresses. Basically, given the lower bound $\delta$ defined in Definition 2, we require a mechanism for adding the nodes to Queue in order of increasing $\delta$ from a query $q$. In case there is some replication in the distributed system, there could be two or more nodes $N_{j} \in \mathcal{N}$ for which $\delta\left(N_{j}, x\right)=0$. However, we only need to find one of them.
When $\mathcal{N}_{x} \neq \emptyset$, Assumption 2 means that the distributed system must be able to search for the next most promising node $\left(N_{n}\right)$ given that we already know a set of nodes $\left(\mathcal{N}_{x}\right)$ which are more, or equal, promising (by considering $\delta$ ) than the next one (i.e., $\mathcal{N}_{x}$ is downward closed).

The role of the downward closed subset $\mathcal{N}_{x}$ will be clarified in the next section which will extensively discuss the algorithm. However, we can anticipate that, because of the algorithm definition, it is a subset of the nodes that, at any given time during the algorithm execution, have been already asked for a local-INN execution. In particular, if $\mathcal{N}_{x}=\emptyset$, Assumption 2 means that any node $N_{c} \in \mathcal{N}$ must be able to find (using some routing mechanism provided by the distributed system), a node $N_{n} \in \mathcal{N}$ for which the distance $\delta\left(N_{n}, x\right)$ is minimum.

If, for a specific data structure, it is not possible to evaluate the lower bound $\delta$, we can consider $\delta\left(N_{i}, q\right)=0$ for every node $N_{i} \in \mathcal{N}$. In this case the order in which the nodes are added to Queue is undefined. However in this case, we will involve all the nodes in (almost) every execution of the DINN algorithm. In fact, given that there is not a lower bound for the distance between the objects stored in a given node and the query, we can not exclude any node a priori.

Please note that, we do not suppose that in the distributed system there is a global knowledge of the network. We only assume that there is a way (usually a routing mechanism) to find the most promising node for the algorithm progress. It can also be noted that, if $\delta$ is strong, the first node added to Queue is the node $N_{n}$ that would contain $x$ (i.e., $\delta\left(N_{n}, x\right)=$ $0)$. Therefore, in this case, the problem of finding the most promising node becomes similar to the lookup problem in DHTs.

While Assumption 2 is the most generic one, there are simpler assumptions that can substitute it. In fact, in Section 4.3.3, we illustrate two sufficient conditions for Assumption 2. Condition 1 guarantees that the next most promising node is always in Queue by just collecting information about neighbors of yet involved nodes. On the other hand, Condition 2 is easily satisfied by data structures able to perform similarity search because it basically makes use of the capability of a system to perform range queries.

\subsection{The algorithm}

In this section we present the definition of our DINN algorithm for retrieving objects in order of decreasing similarity with respect to a given query $q$. In particular, we will define the process of retrieving the next closest object to $q$ at each DINN invocation. In Subsection 4.4 we will present a message reduction optimization in case we want to retrieve more than one object at each DINN invocation. Finally in Subsection 4.5 the proposed algorithm will be extended to parallelize the operations made by distinct nodes.

To perform the $D I N N$ we need to define a node that takes the role of coordinating node $\left(N_{c}\right)$. A good candidate for this role is the initiating node (i.e., the node requesting the search). Another good candidate, in case $\delta$ is strong (see Definition 2) is the node that would store the query (i.e., $\left.\delta\left(N_{c}, x\right)=0\right)$. However, the definition of our DINN algorithm is independent on the particular choice of the coordinating node. This choice only affects the number of messages exchanged during the query execution.

As in [10] we need a Queue (see Definition 1) in which el- 


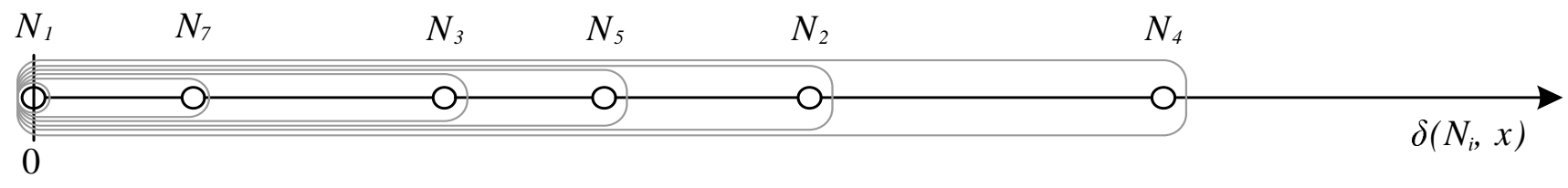

Figure 1: Downward closed set of nodes with respect to object.

ements are ordered according to their key (see Definition 5). Moreover, when an object and an element have the same key, the object comes before the node in Queue. In Queue nodes will be assigned a different key $(\vartheta)$ depending on whether they have already returned objects or not. Thus, we will use the following notation:

Notation 1. $\mathcal{N}^{*} \subset \mathcal{N}$ is the set of nodes that already performed a local-INN.

An important part of the DINN algorithm definition is the definition of the keys used to order elements in Queue.

Definition 5. Given a query object $q \in \mathcal{D}$ we define the key $\vartheta$ as:

- $\vartheta_{x}=d(x, q)$, for any object $x \in \mathcal{D}$;

- $\vartheta_{N_{i}}=\delta\left(N_{i}, q\right)$, for any node $N_{i}$ that has not yet been asked for a local-INN (i.e., $N_{i} \notin \mathcal{N}^{*}$ );

- $\vartheta_{N_{i}}=d\left(l_{i}, q\right)$, for any $N_{i} \in \mathcal{N}^{*}$, where $l_{i} \in \mathcal{X}_{i}$ is the last object that $N_{i}$ returned when performing its local-INN.

Note that both keys used for nodes are lower bounds for the distance between the query $q$ and the next result coming from the local-INN invocation on node $N_{i}$.

The DINN algorithm consists of a loop in which:

1. If Queue is empty, the closest node $\left(N_{n}\right)$ to $q$ that has not yet performed a local-INN is added to Queue. In case $N_{n}$ does not exist, the DINN terminates (there are no more objects in the distributed data structures);

2. Else, if the first element in Queue is a node $\left(N_{i}\right)$, this node is asked to perform a local-INN. Then the returned result $l_{i} \in \mathcal{X}_{i}$ is added to Queue and the key of $N_{i}$ is updated with $\vartheta_{N_{i}}=d\left(l_{i}, q\right)$. In case $N_{i}$ did not return any object (i.e., it has already returned all its objects), the $N_{i}$ is removed from Queue;

3. Else, if the first element in Queue is an object $x$ : let $N_{n}$ be the closest node to $q$ that has not yet performed a local-INN and has $\delta\left(N_{n}, q\right)<d(x, q)$; if $N_{n}$ exists, add it to Queue, otherwise the loop is exited returning $x$ as the next result. Note that if $\mathcal{N}^{*}$ is downward closed $N_{n}$ can be found because of Assumption 2. We prove $\mathcal{N}^{*}$ to be downward closed in Corollary 1 (Section 4.3.1).

Queue must be kept alive for future request of more results. Obviously, the requester can close the session asserting that no more results will be asked. In this case Queue can be discarded.

In Algorithm 1 we give a definition of the DINN algorithm using a pseudo language. The functions and procedures used in Algorithm 1 are defined as follows:

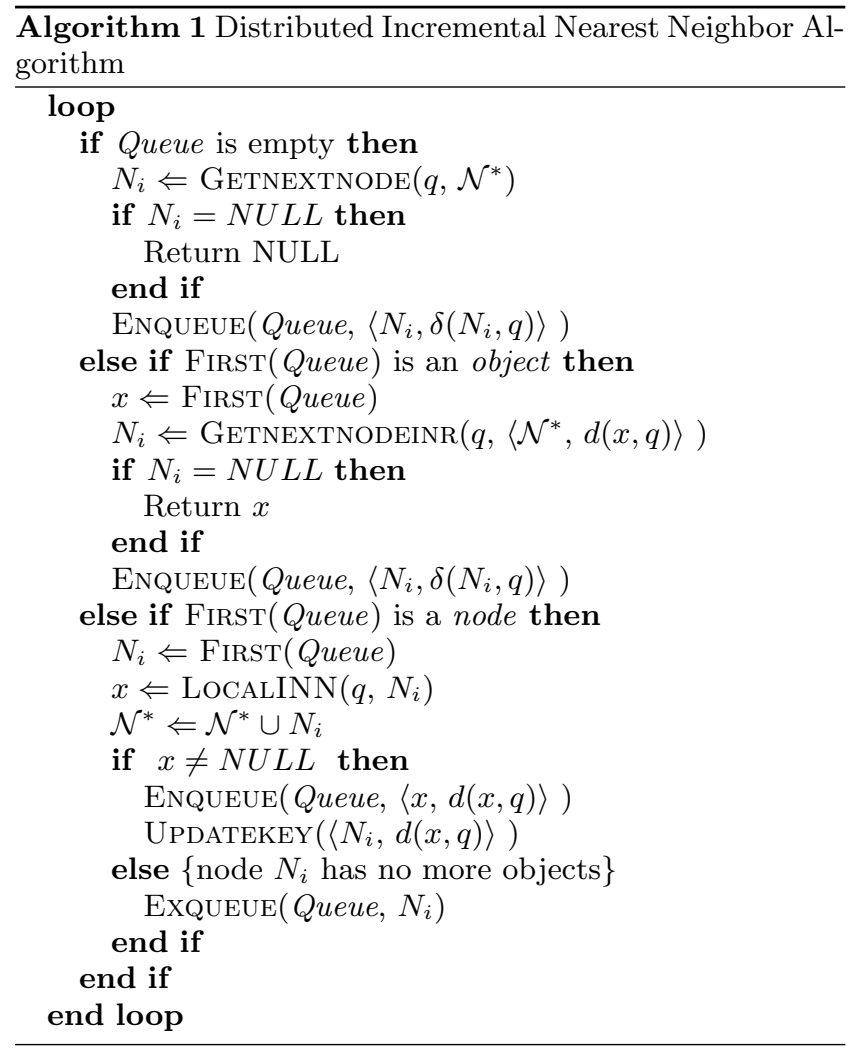

- First(Queue): returns the first element in Queue.

- LocalinN $\left(q, N_{i}\right)$ : asks node $N_{i}$ to return the next result according to its local-INN with respect to the query $q$.

- $\operatorname{Enqueue}(Q u e u e,\langle e, \vartheta\rangle)$ : adds element $e$, either an object or a node, to Queue with key $\vartheta$.

- Updatekey $\left(Q u e u e,\left\langle N_{i}, r\right\rangle\right)$ : updates the key of node $N_{i}$ in Queue with the value $r \in \mathbb{R}^{+}$.

- $\operatorname{Exqueue}(Q u e u e, e)$ : removes element $e$ and its key from Queue.

- Getnextnodeinr $\left(q, \mathcal{N}^{*}, r\right)$ : returns $\arg \min _{N_{i}}\left\{\delta\left(N_{i}, q\right), N_{i} \in\left(\mathcal{N}_{q, r} \backslash \mathcal{N}^{*}\right)\right\}$

- $\operatorname{Getnextnode}\left(q, \mathcal{N}^{*}\right)$ : returns $\arg \min _{N_{i}}\left\{\delta\left(N_{i}, q\right), N_{i} \in\left(\mathcal{N} \backslash \mathcal{N}^{*}\right)\right\}$

Note that if $\mathcal{N}^{*}$ is always downward closed with respect to $q$, because of Assumption 2 it is possible to implement the 
function $\operatorname{Getnextnode}\left(q, \mathcal{N}^{*}\right)$. We prove this in Corollary 1 (Section 4.3.1). Please note also that GETneXtn$\operatorname{OdeinR}\left(q, \mathcal{N}^{*}, r\right)$ can be implemented using Getnextn$\operatorname{ODE}\left(q, \mathcal{N}^{*}\right)$. On the other side, using GetnextnodeinR, we can realize Getnextnode increasing $r$ until a node is found. However, $\operatorname{GetnextnodeinR}\left(q, \mathcal{N}^{*}, r\right)$ can be more efficiently implemented considering that it does not need to return a node if it is farther away than $r$ from $q$.

In Figure 2 we give an example of Queue at a given time during the DINN execution. The dotted lines show from which node every object comes from. Let us suppose that we are searching for the next nearest object to the query $q$ and we have already found some results which are no more in Queue (please note that whenever a result is found it is moved out of Queue). The next element in Queue is $N_{3}$. Thus, we have to to invoke its the $N_{3}$ local-INN to retrieve the $N_{3}$ next result. Let $w$ be the next result retrieved by $N_{3}$. Once $w$ is retrieved, both $w$ and $N_{3}$ are put in the Queue with the same key $d(w, q)$. However, because $w$ is an object, it will be before $N_{3}$ in Queue. If $d(w, q)<d(z, q)$ then $w$ is the first element in Queue and thus it is also the next result of the DINN. Otherwise $z$ is the first element in Queue and also the next result.

\subsubsection{Correctness}

In this section we prove that our DINN algorithm is correct, i.e., it returns objects in order of increasing distance (decreasing similarity) from the query $q$ (Theorem 1 ).

First of all, to guarantee that it is possible to define GETNEXTNODEINR and GETNEXTNODE for a given distributed system under Assumption 2, we must prove that $\mathcal{N}^{*}$ is always downward closed with respect to $q$ :

Corollary 1. At any time during the DINN algorithm execution, the set of nodes $\mathcal{N}^{*}$ (i.e., the set of nodes that already performed a local-INN) is downward closed with respect to the query $q$.

Proof. We prove the corollary using induction. When the algorithm starts Queue is empty and a node $N_{i}$ is added to Queue using $\operatorname{Getnextnode}(q, \emptyset)$ (usually $\delta\left(N_{i}, q\right)=0$ ). After $N_{i}$ has been asked for a result, $\mathcal{N}^{*}$ contains only $N_{i}$ and is downward closed by definition of Getnextnode. At any given time during the algorithm execution, let $N_{n}$ be the node, if it exists, returned either by the function GETNEXTN$\operatorname{OdEINR}\left(q, r, \mathcal{N}^{*}\right)$ or by the function $\operatorname{Getnextnode}\left(q, \mathcal{N}^{*}\right)$. Because of the functions definitions, if $N_{n}$ exists, there is no other node $N_{j} \in \mathcal{N}^{*}$ for which $\delta\left(N_{j}, q\right)<\delta\left(N_{n}, q\right)$. Then $\left(\mathcal{N}^{*} \cup N_{n}\right)$ is still downward closed with respect to $q$.

TheOREM 1 (Correctness). Let $\mathcal{R}$ be the set of objects already returned by the DINN algorithm. Whenever DINN returns an object $x$ there are no objects nearer to the query:

$$
\forall y \in \mathcal{X}, \quad d(y, q)<d(x, q) \Rightarrow y \in \mathcal{R}
$$

Proof. By definition of $\mathcal{X}$ there must be a node $N_{j} \in \mathcal{N}$ for which $y \in \mathcal{X}_{j}$. Using Definition Definitionnot:qrnodes, $d(y, q)<d(x, q) \Rightarrow N_{j} \in \mathcal{N}_{q, d(x, q)}$. Because of the algorithm definition, $\operatorname{Getnextnodeinr}\left(x, d(x, q), \mathcal{N}^{*}\right)$ did not return any node. Then, by GetnextnoDEINR definition, $\left(\mathcal{N}_{q, d(x, q)} \backslash \mathcal{N}^{*}\right)=\emptyset$ and then $N_{j} \in \mathcal{N}^{*}$ (i.e., $y$ belongs to a node which has already been asked for a local$I N N)$. If $N_{y} \in \mathcal{N}^{*}$ has some not returned objects by algorithm definition $N_{j}$ is in Queue with key $d\left(l_{i}, q\right)$ (where $l_{i} \in \mathcal{X}_{i}$ is the last object it returned). Because $x$ is first, $d\left(l_{i}, q\right) \geq d(x, q)>d(y, q)$. Then $y$ must be between the objects $N_{i}$ already returned, which are either in $\mathcal{R}$ or in Queue. But $y$ can not be in the priority because $x$ is first and objects are ordered according to their distance from the query, then $y \in \mathcal{R}$.

\subsubsection{Optimality}

In this section we prove that our DINN algorithm is optimal in terms of number of involved nodes (Theorem 2) and number of local-INN invocations (Theorem 3).

THEOREM 2. The DINN is optimal with respect to the number of involved nodes given the lower bound $\delta$.

Proof. The theorem can be rewritten as follows. Let $\mathcal{N}^{*}$ be the set of involved nodes, $x \in \mathcal{X}$ the last object returned by the $D I N N$ and $q \in \mathcal{D}$ the query object. Whenever the local-INN of $N_{i}$ is invoked, the lower bound $\delta$ of the distance between $q$ and the objects in $N_{i}$ is less than the distance between $q$ and $x$, i.e.,

$$
N_{i} \in \mathcal{N}^{*} \Rightarrow \delta\left(N_{i}, q\right) \leq d(x, q)
$$

Because of the algorithm definition (see Algorithm 1), the last returned object $x$ was at the head of Queue and each node is requested to perform a local-INN result only when they are at the head of Queue. Because of $\delta\left(N_{i}, q\right)$ and $d(x, q)$ are used as key for not yet involved nodes and objects respectively (see Definition 5), the last equation always holds. In fact, both objects and nodes are ordered in Queue according to their keys.

THEOREM 3. The DINN is optimal with respect to the number of local-INN invocations given the lower bounds $\delta$ for the not yet involved nodes, and $d\left(l_{i}, q\right)$ for the yet involved nodes.

Proof. In Theorem 2 we proved that the DINN is optimal in terms of number of involved nodes. Thus, DINN is optimal in terms of local-INN first invocations. Moreover, being $\vartheta_{N_{i}}=d\left(l_{i}, q\right)$ the key (used to order the elements in Queue) for a node $N_{i}$ that already performed a local-INN (see Definition 5), whenever $N_{i}$ is asked to retrieve its next result (using its local-INN) we are sure that the DINN next result will be further away than $d\left(l_{i}, q\right)$. In fact, we are using as key in Queue $d(x, q)$ for every object $x$ and a lower bound for $d\left(y_{i}, q\right)$ for every node $N_{i}$ (see Definition 5 ).

\subsubsection{Sufficient Conditions for Assumption 2}

In this section we give two conditions which are sufficient for Assumption 2. Condition 1 guarantees that the next most promising node is always in Queue just collecting information about neighbors of yet involved nodes (i.e., without generating more messages) and is satisfied by $M C A N$ which we used in our experiments. On the other hand, Condition 2 makes use of the capability to perform range queries and is thus easily satisfied by data structures able to perform similarity search (as the ones presented in [3] and [4]).

Condition 1. Let $\mathcal{N}_{q}$ be a downward closed set of nodes with respect to an object $q \in \mathcal{D}$. For any given $N_{i} \in \mathcal{N}$, let $\mathcal{N}_{i} \subseteq \mathcal{N}$ be the set of nodes which $N_{i}$ is able to contact directly independently from the execution of the current $D I N N$ algorithm. Let $N_{n} \in \mathcal{N}$ be the closest node to 
the query (according to $\delta$ ) which is not in $\mathcal{N}_{x}$ (as defined in Assumption 2). If $N_{n}$ exists, it is in the union of the set of nodes known by the nodes in $\mathcal{N}_{x}$ :

$N_{n}=\arg \min _{N_{i}}\left\{\delta\left(N_{i}, q\right), N_{i} \in\left(\mathcal{N}_{q, r} \backslash \mathcal{N}_{x}\right)\right\} \in \bigcup\left\{\mathcal{N}_{i}, N_{i} \in \mathcal{N}_{x}\right\}$.

Theorem 4. Condition 1 is sufficient for Assumption 2.

Proof. By Condition $1, N_{c}$ can ask each node $N_{i} \in \mathcal{N}^{*}$ which are the nodes it has knowledge about $\left(\mathcal{N}_{i}\right)$. Sorting the union of them $\left(\bigcup\left\{\mathcal{N}_{i}, N_{i} \in \mathcal{N}_{x}\right\}\right) N_{c}$ is able to find $N_{n}$. Thus, Assumption 2 is satisfied.

Condition 1 basically says that it is always possible to pass from one node $N_{n-1}$ to the next one $\left(N_{n}\right)$ just using the information we found in the previous nodes. The information we need is the knowledge they have about other nodes (typically neighbors). This condition is very useful to efficiently implement GETNEXTNODE and it is satisfied by $M C A N$ which is used in our experiments.

Condition 2. For any given object $q \in \mathcal{D}$ and $r \in \mathbb{R}^{+}$, every node $N_{i} \in \mathcal{N}$ is able to know all the nodes (their addresses) in $\mathcal{N}_{x, r}$.

Theorem 5. Condition 2 is sufficient for Assumption 2.

Proof. By Condition 2, $N_{c}$ can ask for all the nodes in $\mathcal{N}_{q, r}$. If $\left(\mathcal{N}_{q, r} \backslash \mathcal{N}_{x}\right) \neq \emptyset$, the next node $N_{n}$ is the nearest to the query in $\left(\mathcal{N}_{q, r} \backslash \mathcal{N}_{x}\right)$. Otherwise, if $\left(\mathcal{N}_{q, r} \backslash \mathcal{N}_{x}\right)=\emptyset$, $N_{c}$ can try again increasing $r$ until $r \leq d_{\max }$. In this last case $N_{n}$ does not exist

Please note that all distributed data structures able to perform a range query, should be able to satisfy Condition 2 (and then Assumption 2). Under Condition 2 GetnextnODEINR is efficiently implemented while GETNEXTNODE can be realized increasing $r$ until either a node is found, using GetnextnodeinR, or $r$ exceeds the max possible value of $d$ (i.e., $\left.d_{\max }=\max (d(y, x), y, x \in \mathcal{D})\right)$.

\subsubsection{Considerations}

The major differences between our DINN algorithm and the INN defined in [10] are:

- Once a node comes at the head of the queue we don't ask it to return all its objects ordered according to their distances from the query. This would be the natural extension for the $I N N$ algorithm, but, in a distributed environment, such an algorithm could not be scalable. Therefore, we ask it to return its next object using its local-INN;

- Whenever a node returns an object, we move it back in the queue using $d\left(l_{i}, q\right)$ as new key ( $l_{i}$ is the last object the $N_{i}$ returned as a result). Please note that $d\left(l_{i}, q\right)$ is a lower bound for the distance between $q$ and the next result coming from the local-INN of $N_{i}$;

- The original $I N N$ algorithm requests a consistency condition (Definition 1 of [10]) to ensure that once a node reaches the head of the queue no other nodes can return objects with a distance smaller than the head node key. This condition has been defined for hierarchical data structure thus limiting the use of their INN algorithm. In our DINN we replaced the consistency condition with Assumption 2.

\subsection{Message reduction}

In this section we give an extension of our DINN to reduce the number of messages when we want to retrieve the next $k^{+} \geq 1$ objects. The price to be paid for the messages reduction is the possibility to ask a node to retrieve more objects than what is strictly necessary. At any given time during the execution of the $D I N N$ :

Notation 2. let $\bar{k}$ be the number of objects already retrieved by the previous invocations of the DINN,

Notation 3. let $k^{+}$be the number of more objects we want to retrieve, and

Notation 4. let $k_{a n s} \leq k^{+}$be the number of results already found by the $D I N N$ during the current invocation.

If a node $N_{i}$ is first in Queue we ask this node to retrieve the next $\hat{k}$ results where:

$$
\hat{k}=k^{+}-k_{a n s}
$$

Because $\hat{k}$ represents the number of objects we need to end the given task (i.e., retrieving the next $k^{+}$objects) we are sure that we will never involve $N_{i}$ again before the current task will be completed. Note that, by definition, $\hat{k} \geq 1$ always holds until the current task is completed.

Furthermore, we can reduce the number of unnecessary objects retrieved, by considering the distance of the $\hat{k}$-th object, if it exists, in Queue.

Definition 6. At any given time during the DINN algorithm execution, let $x_{\hat{k}} \in \mathcal{X}$ be the $\hat{k}$-th object, if it exists, in Queue. To guarantee that node $N_{i}$ will be involved only once during the current task, we ask node $N_{i}$ to perform a sequence of local-INN invocations until at least one of the following conditions is true:

- $\hat{k}$ more objects have been retrieved $\left(\hat{k}=k^{+}-k_{a n s}\right)$;

- $d\left(l_{i}, q\right) \geq d\left(x_{\hat{k}}, q\right)$, where $l_{i}$ is the last object retrieved;

- all the objects stored in $N_{i}$ have been retrieved.

The results coming from $N_{i}$ are added to Queue. If all the objects stored in $N_{i}$ have been retrieved $N_{i}$ is removed from Queue, otherwise its key is update with $\vartheta_{N_{i}}=d\left(l_{i}, q\right)$ and then $\vartheta_{N_{i}} \geq d\left(x_{\hat{k}}, q\right)$. At this stage there are two possibilities: either the $\hat{k}$ enqueued objects are before $N_{i}$ or $N_{i}$ is after $x_{\hat{k}}$. In both cases at least $\hat{k}$ objects are before $N_{i}$ in Queue. Thus, we will not involve $N_{i}$ again in retrieving the next $\hat{k}$ results.

In Figure 2 we give an example of Queue at a given time during the DINN execution. The dotted lines show from which node every object comes from. Let us suppose that we are searching for the next $k^{+}=5$ objects and we have already found the next $k_{\text {ans }}=2$ results (they are no more in Queue). We still have to search for the next $\hat{k}=k^{+}-$ $k_{\text {ans }}=5-2=3$ results. The $\hat{k}$-th object $x_{\hat{k}}$ in Queue is $z$. Using the proposed extension, the $D I N N$ will ask node $N_{3}$ to retrieve objects (using its local-INN) until either 3 objects have been found or the last object $l_{3}$ retrieved by $N_{3}$ has distance $d\left(l_{3}, q\right) \geq d(z, q)$. 


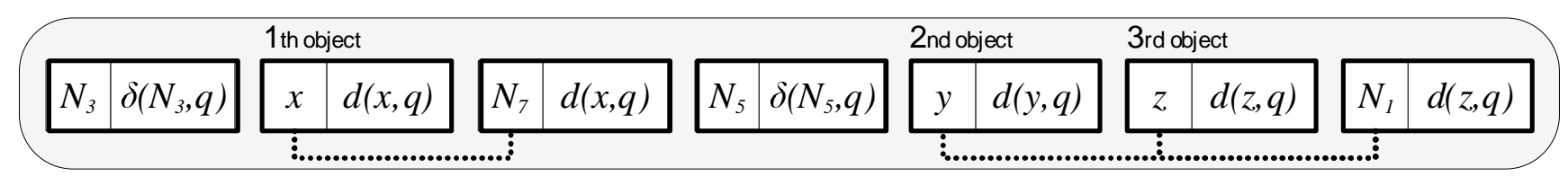

Figure 2: Snapshot of the priority queue at a given time during the execution of the DINN algorithm

\subsection{Parallelization}

The DINN algorithm presented in Section 4.3 always involves only the most promising node - the first in Queue. In this section we give a parallelized version of our DINN.

Generally speaking, the $k-N N$ operation, is not an easily operation to parallelize as the RangeQuery is. To execute a RangeQuery, every single node can perform the search among his objects without considering the results coming from other nodes. Given the query and the range, each node can search among his objects regardless the results found in other peers. To parallelize the DINN algorithm we must accept the possibility to ask a node to give its next result even if it could be not necessary. Furthermore, in a parallelized $D I N N$ it is possible to involve nodes which would not be involved by the serial execution.

Let us assume that at a given time during the algorithm execution $x_{1}$ is the first object in Queue. In principle it is possible that we will ask all the nodes before $x_{1}$ in Queue to invoke their local-INN (e.g., if all these nodes return results further away from $q$ than $x_{1}$ ). To parallelize the DINN execution, we can decide to ask all the nodes before $x_{1}$ to retrieve the next object.

We now give a definition of DINN parallelization which can be also used in combination with the message optimization given in Definition 6 .

Definition 7. Let $x_{\hat{k}} \in \mathcal{X}$ be the $\hat{k}$-th object in Queue and $d\left(x_{\hat{k}}, q\right)$ its distance from the query. Let $p \in[0,1]$ be the parallelization parameter. We parallelize the DINN asking all the nodes $N_{i} \in$ Queue whose $\vartheta_{N_{i}} \leq p d\left(x_{\hat{k}}, q\right)$. In other words, using Definition 5 , a node $N_{i} \in$ Queue is involved iff:

- $\vartheta_{N_{i}}=\delta\left(N_{i}, q\right) \leq p d\left(x_{\hat{k}}, q\right)$,

in case $N_{i} \in \mathcal{N} \backslash \mathcal{N}^{*}$ (i.e., $N_{i}$ has not yet been asked for a local-INN);

- $\vartheta_{N_{i}}=d\left(l_{i}, q\right) \leq p d\left(x_{\hat{k}}, q\right)$, otherwise (i.e., $N_{i} \in \mathcal{N}^{*}$ ) where $l_{i} \in \mathcal{X}_{i}$ is the last object that $N_{i}$ returned invoking its local-INN.

Any involved node is asked to retrieve its next object invoking its local-INN. However, using the DINN optimization for $k-I N N$ search (see Definition 6), any node can be asked to perform more than one local-INN with a single message. However, in this case, there are nodes that are not at the top of Queue, asked to retrieve objects. We can then consider the case in which there are objects before them in Queue. Let $\tilde{k}_{N_{i}}$ be the objects in Queue before node $N_{i}$. The max number of objects we are interested in retrieving from $N_{i}$ is no more $\hat{k}$ but $\hat{k}-\tilde{k}_{N_{i}}$.

In Figure 2 we give a snapshot of Queue at a given time during the DINN execution. As said before, the dotted lines show from which node each object comes from. As before, let us suppose that we are searching for the next $k^{+}=5$ objects and we have already found the next $k_{a n s}=2$ results.
We still have to search for the next $\hat{k}=3$ results. Using the proposed extension, the $D I N N$ will ask node $N_{3}, N_{5}$ and $N_{7}$ to invoke their local-INN and they all will work in parallel. If we also use the message reduction optimization, $N_{3}$ will be asked to retrieve at most 3 objects, while $N_{5}$ and $N_{7}$ will be asked to retrieve at most 2 objects. All of them will stop the iteration of their local-INN if $d(l, q) \geq d(z, q)$, where $l$ is the last object they retrieved.

Unfortunately, there could be some nodes $\left(N_{i}\right)$ not yet in Queue for which $\vartheta_{N_{i}} \leq p d\left(x_{\hat{k}}, q\right)$. In fact, the DINN algorithm does guarantee only that the next most promising node is present in Queue before asking to the first node in Queue to perform a local-INN. In this case the DINN algorithm will continue to be correct, but the parallelization would be reduced. To better parallelize the DINN algorithm is useful to put more nodes in Queue than necessary. As said before, parallelizing the $D I N N$ can increase the total cost. For this reason a parametrized parallelization is useful to find the desired trade-off between total and parallel cost.

Definition 8. Let $\hat{k} \in \mathbb{N}^{+}$, and $x_{\hat{k}} \in \mathcal{X}$ the $\hat{k}$-th object, if it exists, in Queue which is, by definition, ordered. Let $p \in[0,1]$ be the parallelization parameter. We ask all the nodes in Queue whose $\vartheta \leq p d\left(x_{\hat{k}}, q\right)$ until at least one of the following conditions is true (as in Definition 6):

- $\hat{k}$ more objects have been retrieved $\left(\hat{k}=k^{+}-k_{a n s}\right)$;

- $d\left(l_{i}, q\right) \geq d\left(x_{\hat{k}}, q\right)$, where $l_{i}$ is the last object retrieved;

- all the objects stored in $N_{i}$ have been retrieved.

Note that, since $\hat{k} \leq k^{+}$, the degree of parallelization does depend on $k^{+}$. In other words, the more objects we request at each invocation of the DINN algorithm, the greater degree of parallelization we obtain with the same $p$.

In case $x_{\hat{k}}$ does not exist (i.e., there are less than $\hat{k}$ objects in Queue), we involve just the first node (which is at the top of Queue). Once $x_{\hat{k}}$ apeears in Queue, the parallelization is used again.

Another choice, in case $x_{\hat{k}}$ does not exist, is to use, in place of $d\left(x_{\hat{k}}, q\right)$, the distance from the query of the last object in Queue. In this case the operation would became more parallel but also more expensive considering its total cost. The degree of parallelization of the $D I N N$ is also related to the number of nodes present in Queue. Thus, it is important to have more than only the next most promising node $N_{n}$ (see Assumption 2) in Queue. Different strategies can be used to efficiently put nodes in Queue depending on the specific data structure that is used. In our implementation of the DINN over the MCAN, we decided to put in Queue the neighbors of every involved node.

\section{DINN OVER MCAN}




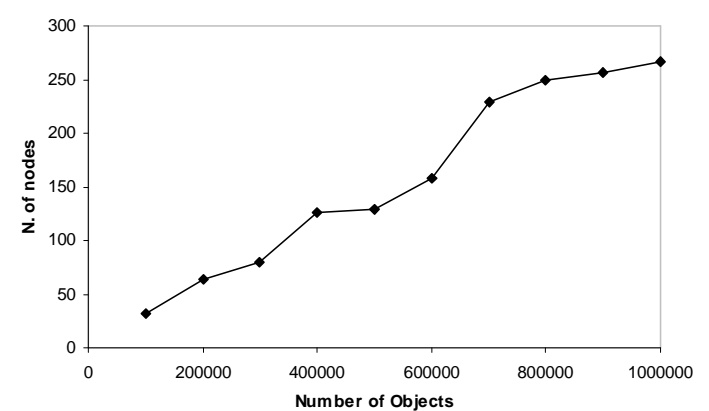

Figure 3: N. of nodes as dataset grows

The $M C A N[8,9]$ is a scalable distributed similarity search structure for metric data. Extending the Content-Addressable Network (CAN), which is a well known Distributed Hash Table, $M C A N$ is able to perform distributed similarity searches between objects assuming that the objects, together with the used distance, are metric. For a complete description of $M C A N$ see [8]. A comparison of $M C A N$ with similar distributed similarity search structure for metric data can be found in [3].

$M C A N$ satisfies Condition 1 which guarantees Assumption 2 as demonstrated in Theorem 4 (see Section 4.3.3). In fact, it can be proved that in $M C A N$ if a node $N_{i}$ is neighbor of a node $N_{j}$ that is closer to the query than $N_{i}$ and $\delta\left(N_{j}, q\right)>0$, then $N_{j}$ is also neighbor of at least one other node which is closer to the query than $N_{j}$. In other word $M C A N$ satisfies Condition 1 and thus also Assumption 2. In fact, given a set of nodes $\mathcal{N}^{*}$ downward closed with respect to $q$, the node $N_{n}$ is always between the neighbors of at least a node $N_{j} \in \mathcal{N}^{*}$ (Theorem 4).

\subsection{Experimental Results}

Experiments have been conducted using a real-life dataset of 1,000,000 objects using real nodes in a LAN network. Each object is a 45-dimensional vector of extracted color image features. The similarity of the vectors was measured by a quadratic-form distance [13]. The same dataset has been used for $[9,3,12,1,4]$. The dimensionality used for the $M C A N$ is 3 as in [9]. All the presented performance characteristics of query processing have been taken as an average over 100 queries with randomly chosen query objects.

To study scalability with respect to the number of objects, we limited the number of objects each node can maintain (the same has been done in $[2,8,9,3,12,4]$ ). When a node exceeds its space limit it splits by sending a subset of its objects to a free node that takes the responsibility for a part of the original region. Note that, limiting the number of objects each node can maintain, we simulate the simultaneous growing of dataset and number of nodes. In Figure 3 we show the number of nodes as the dataset grows.

The parallelization and the number of messages reduction are tuned varying respectively parameter $p$, defined in Definition 8 , and $k^{+}$(i.e., the objects requested at each invocation of the DINN algorithm). As described in Subsection 4.4 , the more the objects $\left(k^{+}\right)$we request at each invocation, the greater degree of parallelization we obtain with the same $p$.

Usually evaluation methodologies of metric space access

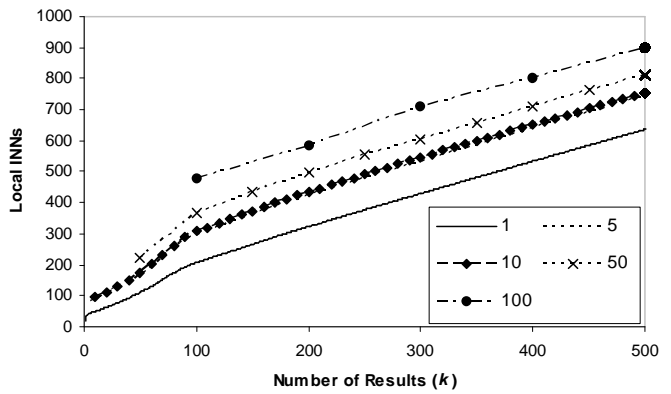

Figure 4: N. of local-INN invocations for different $k^{+}$(parallelization parameter $p=0$ )

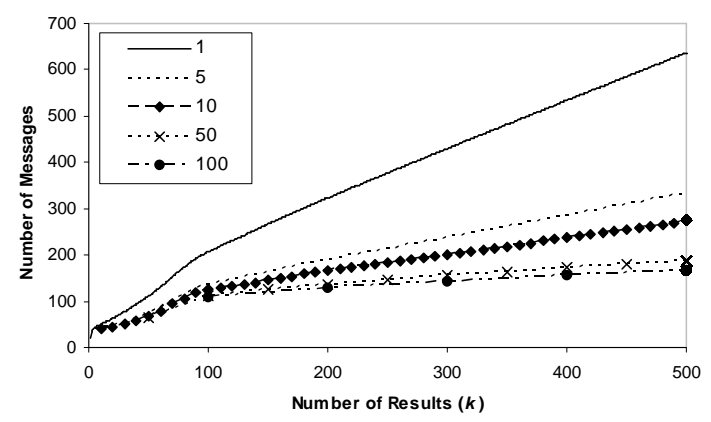

Figure 5: N. of messages for different $k^{+}(p=0)$

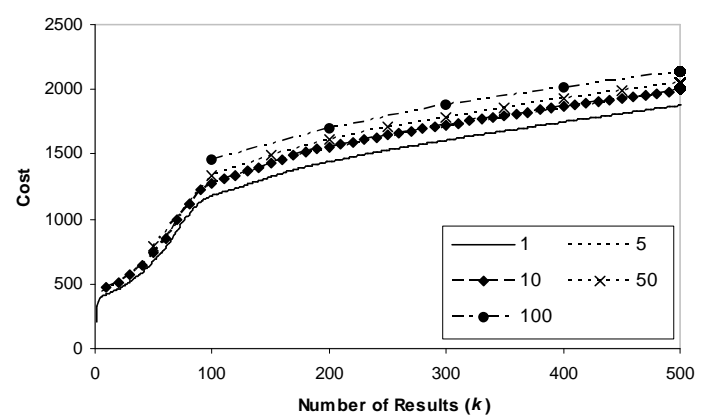

Figure 6: Estimated cost for different $k^{+}(p=0)$ 
methods are based on the number of distance computations. However, to give a fair performance evaluation, we base our evaluation on the number of local-INN invocations. This evaluation approach has the advantage to be independent from the particular local-INN implementation. Furthermore, different nodes could even have different local-INN implementations. We use the following two characteristics to measure the computational costs of a query:

- total number of local-INNs - the sum of the number of local-INN invocations on all involved nodes,

- parallel computations - the maximum number of localINN invocations performed in a sequential manner during the parallel query processing.

Note that the total number of local-INNs corresponds to the cost on a centralized version of the specific structure while the parallel computations, together with the number of messages, directly effects the response time.

In Figure 4 we show the total number of local-INNs for $p=0$ (i.e., no parallelization) for different $k^{+}$as function of the number of results $k$. Note that, to obtain the same number of results $k$ varying $k^{+}$, we need $\left[k / k^{+}\right] D I N N$ invocations. While increasing $k^{+}$does not seem worthwhile since the total number of local-INNs increases, the advantage of greater $k^{+}$is evident observing the number of messages exchanged during the DINN execution in Figure 5. In fact, as said in Subsection 4.4, increasing $k^{+}$, we can reduce the number of messages.

Since obtaining the first result from a local-INN in an arbitrary node is significantly more expensive than obtaining the next ones, a more realistic approach is to consider the cost of the first result of a local-INN as several times the cost of subsequent local-INN invocations. In Figure 6 we report the same result of Figure 4, but assuming that the first invocation cost of a local-INN is 10 times the cost of subsequent invocations. In this case the gap between the graphs for different $k^{+}$remains but it decreases. Note that, since in this case there is no parallelization, there is no difference between the parallel and total cost.

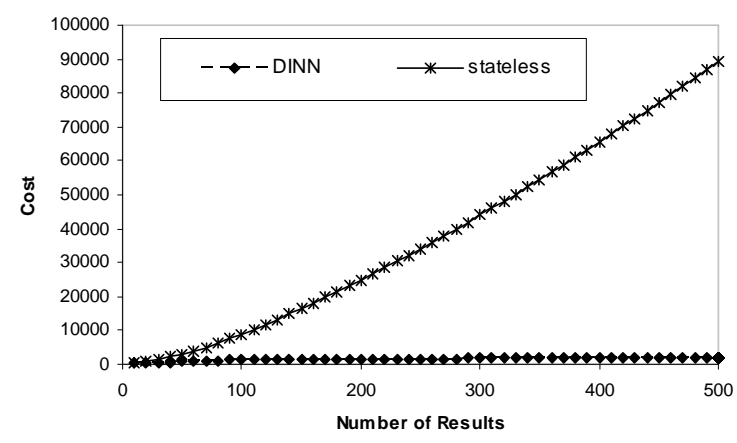

Figure 7: Total estimated costs $\left(p=0, k^{+}=10\right)$

In Figure 7 we show the estimated cost for retrieving up to 500 objects, 10 by 10 (i.e., $k^{+}=10$ ) comparing the defined DINN with a stateless execution of the DINN in which after searching first 10 objects we destroy Queue and then we ask for the next 10 objects (thus requesting a 20NearestNeighbor search from scratch) and so on. Here we
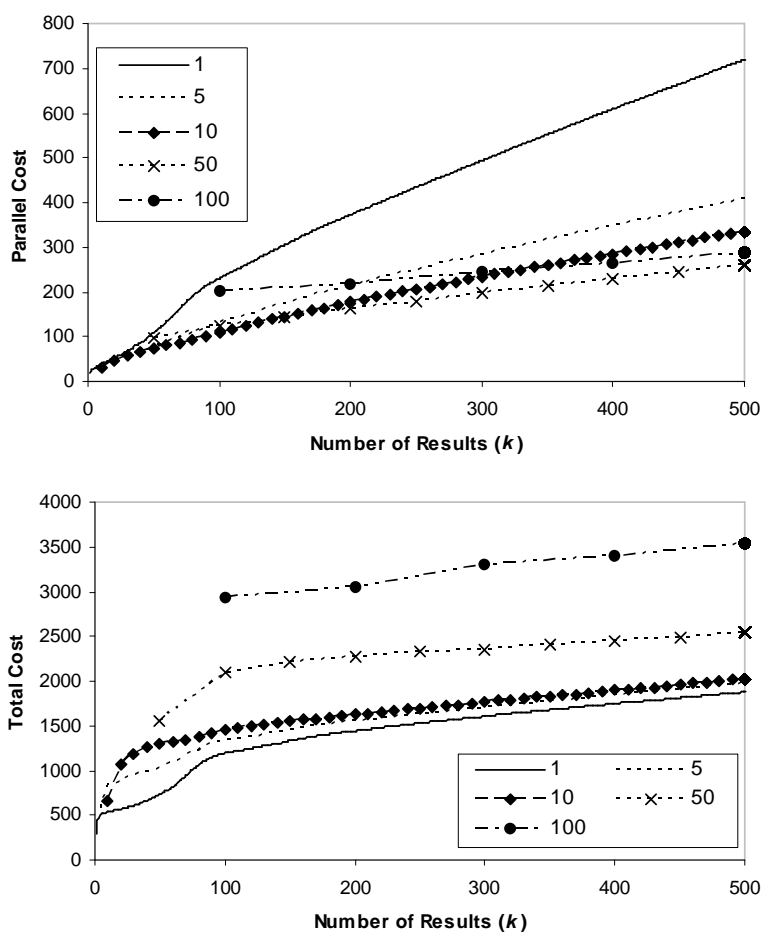

Figure 8: Parallel and total estimated costs for different $k^{+}(p=1)$

want to underline that the use of an Incremental Nearest Neighbor algorithm when the number of desired neighbors is unknown in advance is mandatory to preserve efficiency. In fact the cost of retrieving the next $k^{+}$once a given number of results has already been retrieved using a stateless approach is prohibitive.

Let's now consider the parallelized version of the DINN defined in Subsection 4.5. In Figure 8 we compare the total and parallel cost when $p=1$ (i.e., maximizing the parallelization). The graph of the parallel cost demonstrates the advantage of the parallel execution. Observing for instance $k=100$ for the case $k^{+}=10$, the parallel cost is slightly larger than 100, while for the same case the sequential cost (Figure 6) is about $1300 . k^{+}=10$ seems a good trade off between the total and the parallel cost. In fact, the total cost is almost the same as of the sequential case.

Another set of experiments were conducted by varying $p$ from 0 to 1 for a growing dataset. In this experiments we fixed $k=500$ and used various $k^{+}$.

In Figure 9(a) we report the costs for growing dataset, number of results $k=500$ and $k^{+}=1$. The total cost does not significantly vary with $p$, i.e., parallelization, for $k^{+}=$ 1 , is obtained without increasing the total cost. Another important aspect is that parallel cost is slightly influenced by the dataset size when the parallelization degree is maximum $(p=1)$.

In Figure 9(b) we report the costs for growing dataset, $k=500$ and $k^{+}=10$. We can see that increasing $k^{+}$the differences between the parallel costs of different degree of parallelism $(p)$ are more relevant. However, the total cost for different $p$ are very similar and almost the same of the 

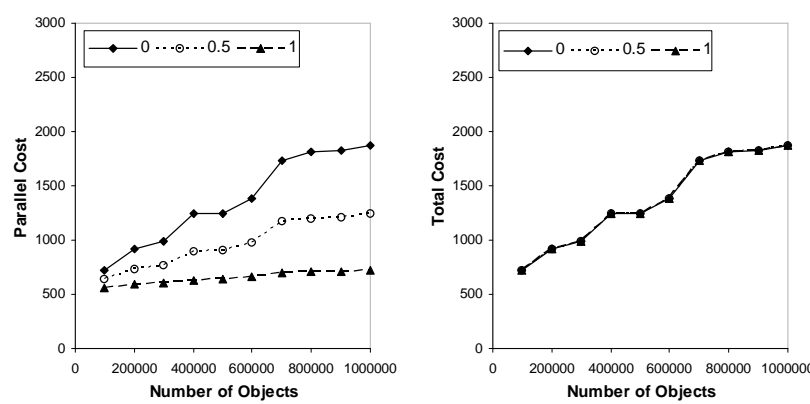

(a) $k^{+}=1$
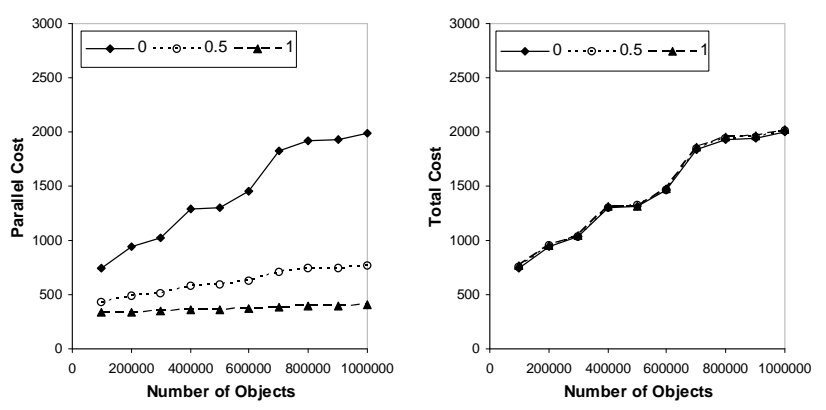

(b) $k^{+}=10$
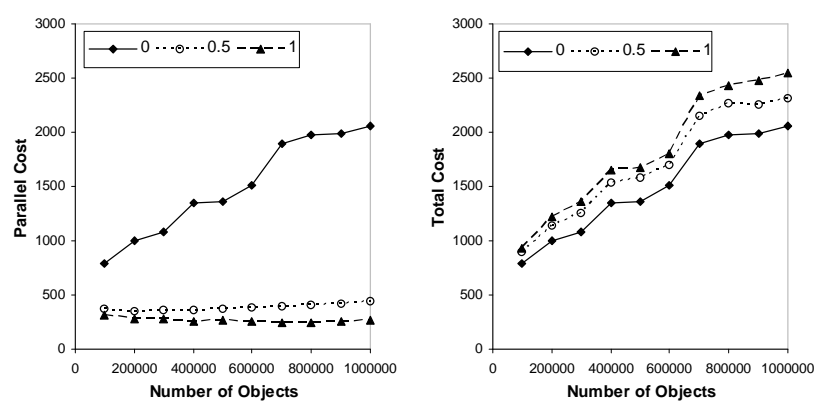

(c) $k^{+}=50$

Figure 9: Parallel and total Estimated Costs for obtaining 500 results for various values of the parameter $p$. Each subfigure reports the result presented obtained using different $k^{+}$

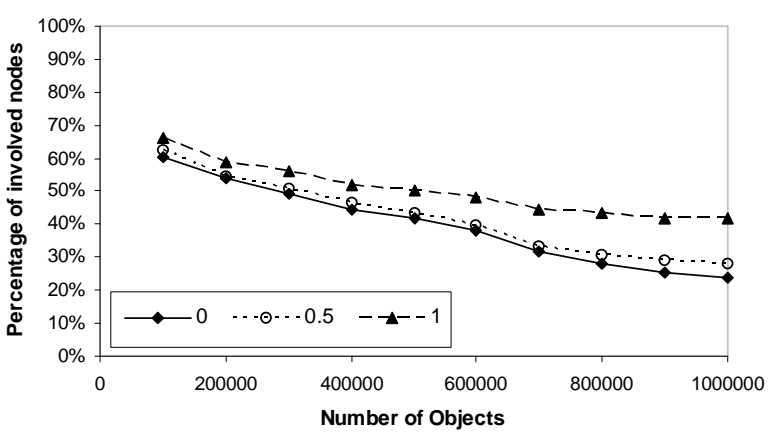

Figure 10: Average percentage of involved nodes for obtaining 50 results for $k^{+}=1$ and various values of the parameter $p$. ones obtained for $k^{+}=1$ in Figure 9(a). It is also important to observe that for $p=1$ the parallel cost scale.

Finally, in Figure 9(c) we report the costs for $k=500$ and $k^{+}=50$. In this case the parallel cost is better than for the $k^{+}=1$ case but the total cost does depend on $p$. However, the most important result is that the parallel cost not only scale with respect to the dataset size, but it slightly decreases. Obviously, this is possible because we are adding more resources (nodes) as the dataset size increase (proportionally), but this should be common in a $\mathrm{P} 2 \mathrm{P}$ environment where typically more nodes means more data and vice-versa.

In Figure 10 we report the percentage of involved nodes for $k^{+}=10$ as the dataset grows. As expected, the more parallelism, the greater percentage of involved nodes. However, it is interesting to notice that results for $p=0.5$ and $p=1$ are almost the same. Considering scalability with respect to the dataset size, it is important that the percentage of involved nodes does decrease with the number of objects, i.e., with the number of nodes.

\section{CONCLUSIONS AND FUTURE WORK}

Distributed incremental nearest neighbor search is a big challenge for at least two reasons. It is quite handy to have a possibility to easily increment the number of nearest neighbors at a low cost instead of being forced to an expensive solution of specifying high values of $k$ to ensure having enough objects in all situations or starting the nearest neighbor search over and over again whenever the value of $k$ grows. Second, distributed environments do not allow application of existing centralized solutions and completely new solutions are needed.

In this paper, we have defined a distributed incremental nearest neighbor especially suitable for structured P2P similarity search networks. The proposed algorithms have been implemented in a large network of computers using $M C A N$ and extensively tested on a real-life data collection: color features of images. We proved our algorithm to be optimal in terms of both the number of involved nodes and the number of local-INN invocations when executed in a serial way. However, our algorithm also allows controlling the degree of parallelism and the number of messages by using two special parameters.

As a next step of our research, we plan to apply this distributed incremental nearest neighbor search to other distributed similarity search structures, such as $G H T^{*}[2], V P T^{*}$ [3], or M-Chord [12]. Naturally, this incremental approach will vitally be important in developing multi-feature similarity search execution strategies which are needed by the top $k$ multi-feature queries.

\section{ACKNOWLEDGMENTS}

This work has been partially supported by the SAPIR (Search In Audio Visual Content Using P2P IR) project, funded by the European Commission under IST FP6 (Sixth Framework Programme, Contract no. 45128) and by the NeP4B (Networked Peers for Business) FIRB project, funded by the Italian Council.

\section{REFERENCES}

[1] Michal Batko, Vlastislav Dohnal, and Pavel Zezula. M-Grid: similarity searching in grid. In P2PIR '06: 
Proceedings, pages 17-24, New York, NY, USA, 2006. ACM Press.

[2] Michal Batko, Claudio Gennaro, and Pavel Zezula. Similarity grid for searching in metric spaces. In 6 th Thematic Workshop of the EU Network of Excellence DELOS. Revised Selected Papers, volume 3664 of $L N C S$, pages 25-44. Springer-Verlag Berlin Heidelberg, 2004.

[3] Michal Batko, David Novak, Fabrizio Falchi, and Pavel Zezula. On scalability of the similarity search in the world of peers. In InfoScale'06: Proceedings, page 20, New York, NY, USA, 2006. ACM Press.

[4] Michal Batko, David Novak, Fabrizio Falchi, and Pavel Zezula. Scalability Comparison of Peer-to-Peer Similarity-Search Structures. Future Generation Computer Systems, to appear.

[5] Ronald Fagin. Combining fuzzy information from multiple systems. Journal of Computer and System Sciences, 58(1):83-99, 1999.

[6] Ronald Fagin, Amnon Lotem and Moni Naor. Optimal Aggregation Algorithms for Middleware. In Proc. ACM Symp. Principles of Database Systems, pages 102-113, 2001.

[7] Fabrizio Falchi, Claudio Gennaro, Fausto Rabitti, and Pavel Zezula. A distributed incremental nearest neighbor algorithm. In InfoScale '07: Proceedings of the Second International Conference on Scalable Information Systems, New York, NY, USA, 2007. ACM Press.

[8] Fabrizio Falchi, Claudio Gennaro, and Pavel Zezula. A content-addressable network for similarity search in metric spaces. In DBISP2P '05: Proceedings, volume 4125 of $L N C S$, pages 98-110. Springer, 2005.

[9] Fabrizio Falchi, Claudio Gennaro, and Pavel Zezula. Nearest Neighbor Search in Metric Spaces through Content-Addressable Networks. Information Processing \& Management, 43(3):665-683, May 2007.

[10] Gísli R. Hjaltason and Hanan Samet. Distance browsing in spatial databases. ACM Transactions on Database Systems (TODS), 24(2):265-318, 1999.

[11] Anirban Mondal, Yi Lifu, and Masaru Kitsuregawa. P2PR-tree: An r-tree-based spatial index for peer-to-peer environments. In EDBT 2004 Workshops. Revised Selected Papers, volume 3268 of LNCS, pages 516-525. Springer-Verlag Berlin Heidelberg, 2004.

[12] David Novak and Pavel Zezula. M-chord: a scalable distributed similarity search structure. In InfoScale'06: Proceedings, page 19, New York, NY, USA, 2006. ACM Press.

[13] Thomas Seidl and Hans-Peter Kriegel. Efficient user-adaptable similarity search in large multimedia databases. In $V L D B$ '97: Proceedings, pages 506-515, San Francisco, CA, USA, 1997. Morgan Kaufmann Publishers Inc.

[14] Ion Stoica, Robert Morris, David Liben-Nowell, David R. Karger, M. Frans Kaashoek, Frank Dabek, and Hari Balakrishnan. Chord: a scalable peer-to-peer lookup protocol for internet applications. IEEE/ACM Transactions on Networking (TON), 11(1):17-32, 2003.

[15] Egemen Tanin, Deepa Nayar, and Hanan Samet. An efficient nearest neighbor algorithm for $\mathrm{p} 2 \mathrm{p}$ settings.
In dg.o2005: Proceedings, pages 21-28. Digital Government Research Center, 2005.

[16] Pavel Zezula, Giuseppe Amato, Vlastislav Dohnal, and Michal Batko. Similarity Search. The Metric Space Approach, volume 32 of Advances in Database Systems. Springer Science + Business Media, Inc., 233 Spring Street, New York, NY 10013, USA, 2006. 